\title{
The intercalation of imidazoacridinones into DNA induces conformational changes in their side chain ${ }^{\star \star}$
}

\author{
Jan Mazerski ${ }^{\bowtie}$ and Karolina Muchewicz \\ Department of Pharmaceutical Technology and Biochemistry, Faculty of Chemistry, Technical \\ University of Gdańsk, G. Narutowicza 11/12, 80-952 Gdańsk, Poland
}

Received: 25 October, 1999

Key words: imidazoacridinones, antitumor drugs, structure of intercalation complex, conformational changes, molecular dynamics simulations, C-1311

\begin{abstract}
Imidazoacridinones (IAs) are a new group of highly active antitumor compounds. The intercalation of the IA molecule into DNA is the preliminary step in the mode of action of these compounds. There are no experimental data about the structure of an intercalation complex formed by imidazoacridinones. Therefore the design of new potentially better compounds of this group should employ the molecular modelling techniques.

The results of molecular dynamics simulations performed for four IA analogues are presented. Each of the compounds was studied in two systems: i) in water, and ii) in the intercalation complex with dodecamer duplex d(GCGCGCGCGCGC) 2 . Significant differences in the conformation of the side chain in the two environments were observed for all studied IAs. These changes were induced by electrostatic as well as van der Waals interactions between the intercalator and DNA. Moreover, the results showed that the geometry of the intercalation complex depends on: i) the chemical constitution of the side chain, and ii) the substituent in position 8 of the ring system.
\end{abstract}

Imidazoacridinones (IAs) are a new group of potent antitumor compounds developed in our Department by a research group headed by Prof. Jerzy Konopa [1-3]. These compounds contain a planar polycyclic acridinone system, which could intercalate to DNA, and a diaminoalkyl side chain at position 5 which can stabilise the drug/DNA complex (Scheme 1).

The biological properties of IAs depend on two structural factors: i) the chemical structure of the side chain, and ii) the presence and

\footnotetext{
${ }^{\text {Presented at the }} 7^{\text {th }}$ International Symposium on Molecular Aspects of Chemotherapy, September 8-11, 1999, Gdańsk, Poland.

* This work was supported by the State Committee for Scientific Research (KBN, Poland) grant No. 3 T09A 12414 and in part by Faculty of Chemistry, Technical University of Gdańsk (Poland).

${ }^{\otimes}$ To whom correspondence should be addressed.

Abbreviations: IA, imidazoacridinone; MD, molecular dynamics; C, and G: deoxynucleotides of cytosine and guanine, respectively.
} 
kind of substituent in position 8. C-1311, has been selected as the most active derivative from the imidazoacridinone group, and is currently undergoing phase I clinical trials [4].
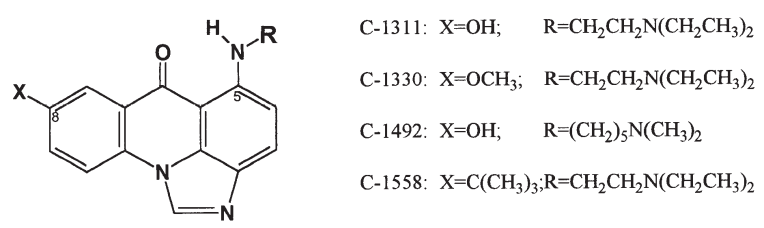

Scheme 1. Chemical structure of imidazoacridinones (IAs) studied.

The intercalation of IAs into DNA is a necessary though not a sufficient condition for the drug activity [5, 6]. It seems that high cytotoxic and antitumor activity of some IAs resulted from their susceptibility to metabolic activation [7]. In a cell free system, IAs bound covalently to DNA after previous enzymatic activation by a horseradish peroxidase $/ \mathrm{H}_{2} \mathrm{O}_{2}$ system [8] This activation may also occur after IA intercalation into DNA.

This observation directly inspired us to undertake the study on the structure of the IA/DNA complexes as well as on interactions responsible for stability of the complex.

There are no experimental data about molecular structure of the IA/DNA complex on the atomic level. Therefore, the application of computer-modelling techniques, such as molecular dynamics (MD) seems to be reasonable. The MD simulations have been increasingly used to study interactions of DNA with various agents [9-17].

In this study we present the results of extensive computer simulations on a dodecamer duplex d(GCGCGC|GCGCGC) 2 with the imidazoacridinone molecule intercalated between central GC base pairs. Four rationally selected IAs were used in this study (Scheme 1). The compounds studied have three different substituents in position 8 and two different side chains. The dynamic behaviour of each IA was simulated in two different systems: i) IA surrounded by about 1700 water molecules, ii) IA intercalated to the dodecamer duplex and surrounded by about 1300 water molecules. The results obtained for the two systems were carefully compared. Any significant difference in the geometry of IA was interpreted as a result of interactions with the dodecamer. Based on the results of simulations obtained for the duplex containing system the most probable structure of the intercalation complexes has been proposed for each IA studied. The influence of the side chain, as well as of the substituent in position 8 on the dynamic behaviour of IA in the two systems studied and on the structure of IA/dodecamer complex is also discussed.

\section{METHODOLOGY}

The dynamics simulations reported in this paper were performed with GROMOS 96 [18], a set of molecular dynamics programs from the University of Groningen, implemented on INDIGO 2 work station. The periodic boundary condition was applied, all bond lengths were constrained to their equivalence values using SHAKE algorithm [19], and distances related to Watson-Crick hydrogen bonds were restrained to $0.21 \mathrm{~nm}$ during all simulations [20]. A dielectric constant of 1 and a cut-off of $0.8 \mathrm{~nm}$ were used for non-bonded interactions, whereas a cut-off of $1.4 \mathrm{~nm}$ was used for long term electrostatic interactions. These cut-off values were proved in previous simulations of this kind to be acceptable and represent a compromise between accuracy and computational efficiency [17, 21]. An appropriate number of counterions, $\mathrm{Cl}^{-}$for IAs and $\mathrm{Na}^{+}$ for dodecamer, and water molecules was added to the system. In order to save computation time the intercalation phenomena were simulated with the DNA fragment in which the intercalation cavity was pre-formed [17].

The crystal co-ordinates of C-1311 atoms (Dzięgielewski, J., Ślusarski, B., Konitz, A., Składanowski, A. \& Konopa, J., unpublished) were used in an initial step of the creation of the simulated system. The initial co-ordinates 
of other IAs studied were generated by molecular modelling of the molecules by program INSIGHT II (MSI) starting from crystal co-ordinates of C-1311. Designations of IAs atoms used in our work are shown on Scheme 2 for C-1311 molecules. dated every 10 steps. The system was coupled to an external thermal and pressure bath of $300 \mathrm{~K}$ and 1 barr, respectively, with time constants equal to 10 fs (for temperature) and $50 \mathrm{fs}$ (for pressure).

$\checkmark$ iv) the system obtained in step iii) was then

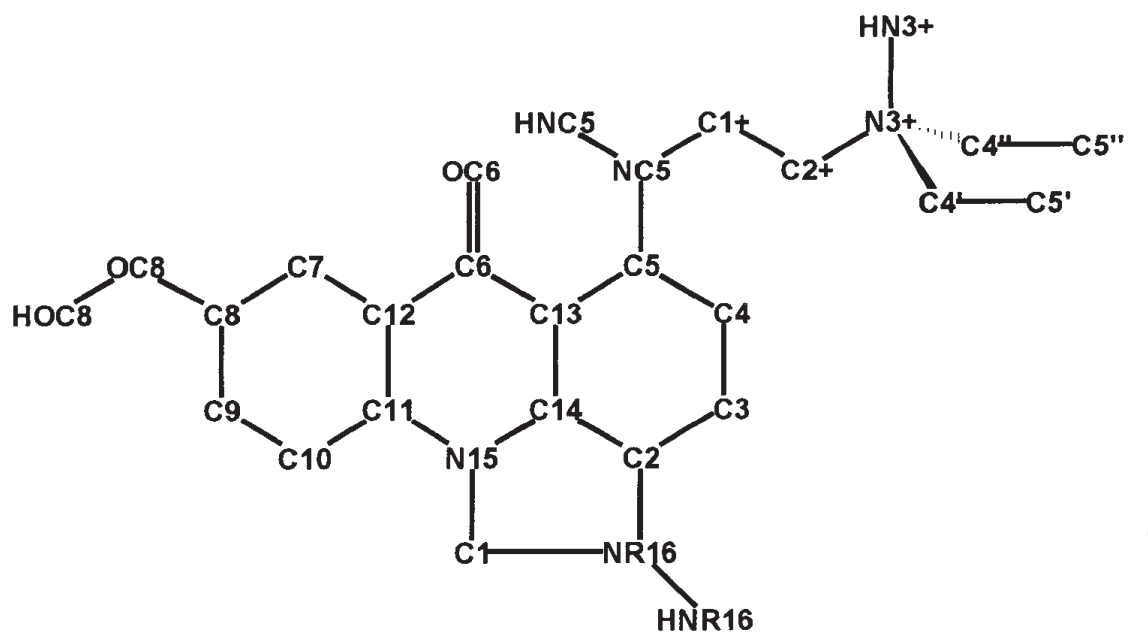

Scheme 2: Designations of C-1311 atoms used in the present work.

For other IAs an analogous system was used.
The following protocol of calculation was applied for each IA:

- ia) for simulations in water a molecule of IA was placed in the middle of a rectangular $3 \times 3 \times 5 \mathrm{~nm}$ box. About 1700 water molecules and $2 \mathrm{Cl}^{-}$counterions were inserted into the box.

$\checkmark$ ib) for simulations of the intercalation complex, the DNA fragment with IA inserted between central base-pairs was placed in the middle of a rectangular $3 \times 3 \times 5 \mathrm{~nm}$ box. About 1400 water molecules, $22 \mathrm{Na}^{+}$, and $2 \mathrm{Cl}^{-}$counterions were inserted into the box.

-ii) 100 steps of the steepest descent minimisation were applied to the system. The list of non-bonded interactions was updated after each step.

-iii) the system was thermalised at $300 \mathrm{~K}$ by $10 \mathrm{ps}$ MD simulation (5000 steps of $2 \mathrm{fs}$ ). The atomic velocities were reassigned according to a Maxwell-Boltzmann distribution at the beginning of the thermalisation. The co-ordinates of IA (and DNA) atoms were constrained to their initial positions. The list of non-bonded interactions was up- relaxed by a $10 \mathrm{ps}$ MD simulation. The atomic velocities were also reassigned at the beginning of this step and the system was coupled to the thermal and pressure bath. An additional potential which restrained distances related to Watson-Crick hydrogen bonds was used in this and all further steps. The list of non-bonded interactions was updated every 20 steps during relaxation and all further simulations.

$\checkmark v$ ) the system prepared in such a way was next simulated for $80 \mathrm{ps}$ with initial reassignment of atomic velocities. The conditions of simulation were the same as for relaxation. The co-ordinates of the system were saved every $0.4 \mathrm{ps}$ and formed trajectories of 200 geometries.

\section{RESULTS}

The overall shape of the IA molecule is mainly determined by geometrical states (torsion angles) of three bonds (Scheme 2):

i) C5-NC5; the state of this bond determines the orientation of the planar aro- 
matic amino group in position 5 and indirectly the orientation of the side chain in relation to the ring system,

$\checkmark$ ii) NC5-C1+; the state of this bond directly determines the orientation of the side chain,

-iii) C8-OC8; the state of this one determines the orientation of the hydroxyl or methoxyl group attached to the ring system in position 8.

The dynamic behaviour of these torsion angles was the main subject of our study.

\section{C-1311}

The comparison of time courses of the torsion angles discussed above, obtained for the two environments studied, revealed some of the characteristic properties of the C-1311 molecule (Fig. 1).

The amino group in position 5 is nearly coplanar with the ring system in the two systems studied (Fig. 1a). However, the rest of the side chain is located out of the plane. This is a consequence of the geometrical state of the bond $\mathrm{NC} 5-\mathrm{C} 1+$ (Fig. 1b). The mean value of this torsion angle is equal to about $237^{\circ}$ for C-1311 in water and to about $130^{\circ}$ for the intercalation complex. A more detailed analysis of the values of this torsion angle showed that the bond may exist in two similar, but somewhat different, geometrical states. These states are characterised by the mean values of the torsion angle equal to about $215^{\circ}$ and $265^{\circ}$, respectively, in water and about $110^{\circ}$ and $145^{\circ}$ in the intercalation complex.

It is noteworthy that the orientation of the side chain in water is stable above all expectations. This may be a result of the presence of stable, intramolecular hydrogen bonds. The analysis of the trajectory revealed that in fact, a set of such bonds exists during the simula-

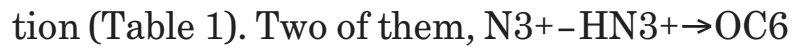
and $\mathrm{N} 3+-\mathrm{HN} 3+\rightarrow \mathrm{NC} 5$, are responsible for stabilisation of the side chain orientation in relation to the ring system. It is interesting to note that the hydrogen atom $\mathrm{HN} 3+$ forms three-center hydrogen bonds during about $13.5 \%$ of the simulation time. The same network of hydrogen bonds is also responsible for stability of the characteristic "closed“ conformation of the side chain (Fig. 2a).

In the case when the C-1311 molecule is intercalated into the dodecamer duplex the geometrical state of the bond $\mathrm{NC} 5-\mathrm{C} 1+$ changes (Fig. 1b) and the side chain forms a new, also

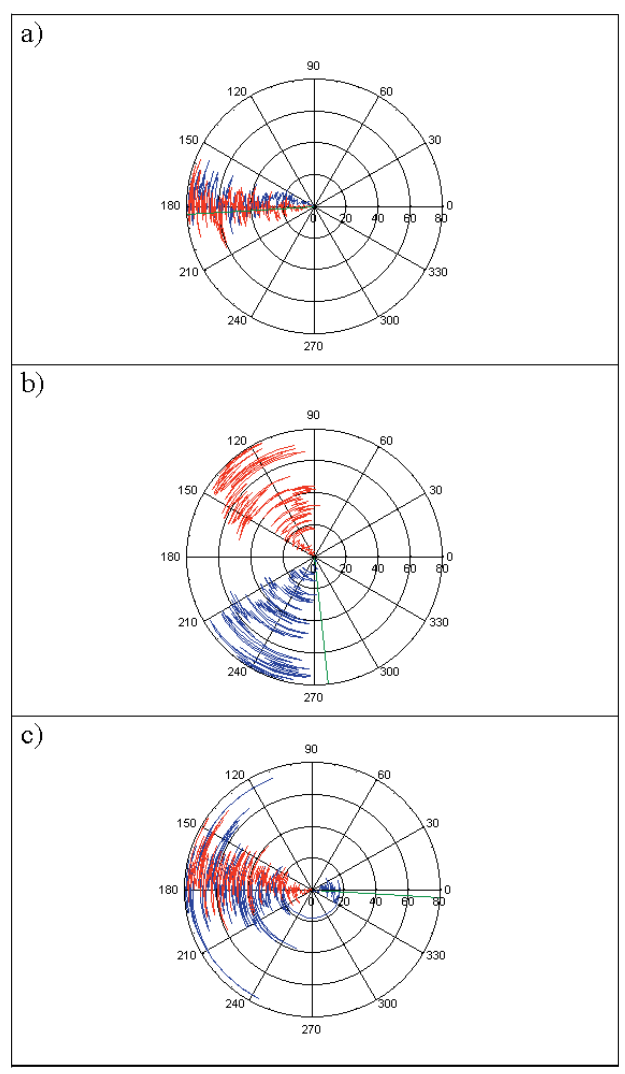

Figure 1. Histories of the torsion angles of selected bonds of C-1311: a) C5-NC5, b) NC5-C1+, c) C8-OC8.

Blue and red lines represent, respectively, values of the torsion angles in water, and in the intercalation complex. Solid green lines represent torsion angles observed in the monocrystal (Dzięgielewski, J., Ślusarski, B., Konitz, A., Składanowski, A. \& Konopa, J., unpublished).

stable, „open“ conformation (Fig. 2b). This conformation is a result of the side chain fitting to the minor groove of the dodecamer (Fig. 3).

During $80 \mathrm{ps}$ of the simulation of the C-1311/dodecamer complex the hydrogen 
Table 1. The hydrogen bonds detected during dynamic simulations

\begin{tabular}{|c|c|c|c|c|}
\hline \multirow{2}{*}{ Hydrogen bonds } & \multicolumn{4}{|c|}{ Compounds } \\
\hline & C-1311 & C-1330 & C-1492 & C-1558 \\
\hline \multicolumn{5}{|l|}{ In water } \\
\hline NC5-HNC5 $\rightarrow$ OC6 & $25.5 \%$ & & $58.5 \%$ & $23.2 \%$ \\
\hline $\mathrm{N} 3+-\mathrm{HN} 3+\rightarrow \mathrm{OC} 6$ & $32.5 \%$ & $78.5 \%$ & & \\
\hline $\mathrm{N} 3+-\mathrm{HN} 3+\rightarrow \mathrm{NC} 5$ & $31.0 \%$ & $43.0 \%$ & & \\
\hline OC6HN3+ $\rightarrow$ NC5 (3-center) & $13.5 \%$ & $35.5 \%$ & & \\
\hline \multicolumn{5}{|l|}{ In the intercalation complex } \\
\hline NC5-HNC5 $\rightarrow$ OC6 & $82.5 \%$ & $75.5 \%$ & $63.5 \%$ & $47.6 \%$ \\
\hline NR16-HNR16 $\rightarrow$ O1P(20C) & $17.0 \%$ & $27.0 \%$ & & $50.8 \%$ \\
\hline $\mathrm{NC} 5-\mathrm{HNC} 5 \rightarrow \mathrm{O} 5 *(7 \mathrm{G})$ & & & & $44.8 \%$ \\
\hline $\mathrm{NC} 5-\mathrm{HNC} 5 \rightarrow \mathrm{O} 3 *(6 \mathrm{C})$ & & & & $20.8 \%$ \\
\hline $\mathrm{N} 3+-\mathrm{HN} 3+\rightarrow \mathrm{O}^{*}(6 \mathrm{C})$ & & & & $27.6 \%$ \\
\hline
\end{tabular}

Numbers in the Table indicated the percentage of simulation time during which a particular hydrogen bond has been observed. The hydrogen-acceptor distance shorter than $0.25 \mathrm{~nm}$ and donor-hydrogen-acceptor angle larger than $120^{\circ}$ were used as the criteria of the existence of a simple hydrogen bond. The sum of angles: donor-hydrogen-hydrogen-acceptor 1, donor-hydrogen-acceptor 2, and acceptor 1-hydrogen-acceptor 2 larger than $350^{\circ}$ was used as the criterium for a three-center hydrogen bond.

atom $\mathrm{HN} 3+$ forms hydrogen bonds with four water molecules. In turn, three of these molecules form hydrogen bonds with phosphate oxygen of $7 \mathrm{G}$ or sugar oxygen $\mathrm{O}^{*}$ of $6 \mathrm{C}$, Fig. 4. The dynamic network of these hydrogen bonds supports the characteristic behaviour of the side chain:

i) stable, out of plane orientation, and

-ii) dynamic transition between two geometrical states of the NC5-C1+ bond (Fig. 1b).

The $-\mathrm{OH}$ group in position 8 is nearly coplanar with the ring system in the two systems studied (Fig. 1c). However, a range of torsion angle values of the bond $\mathrm{C} 8-\mathrm{OC} 8$ is significantly wider in water than in the intercalation complex. It is interesting to note that direct hydrogen bonds between this group and the dodecamer have not been detected. Instead, the 8-OH group is involved in formation of hydrogen bonds with water molecules. Some of these molecules form also hydrogen bonds with the dodecamer (Fig. 4). Thus, these water molecules are involved in formation of "water bridges" between the 8-OH group and the base pairs of the dodecamer duplex.
A direct hydrogen bond between the imidazole ring and a phosphate group of the $20 \mathrm{C}$ is observed during $17 \%$ of the simulation time (Table 1). In addition, the imidazole hydrogen atom participates in formation of a dynamic network of hydrogen bonds with several water molecules. In turn, these water molecules form hydrogen bonds with some oxygen atoms of 17G, 18C, 19G, and 20C (Fig. 4).

\section{C-1330}

A comparison of the dynamic trajectory obtained for compounds C-1311 and C-1330 should visualize the role of hydroxyl and methoxyl group in position 8 in the behaviour of the whole IA molecule. Our dynamic simulations revealed that this small difference in the chemical constitution of the imidazoacridinone system has a significant influence on the dynamic behaviour, shape, and conformation of the molecule.

The side chain of the C-1330 molecule exhibits dynamic properties similar to those of C-1311 (Fig. 5 a, b). A small but significant difference could be observed only for the geomet- 

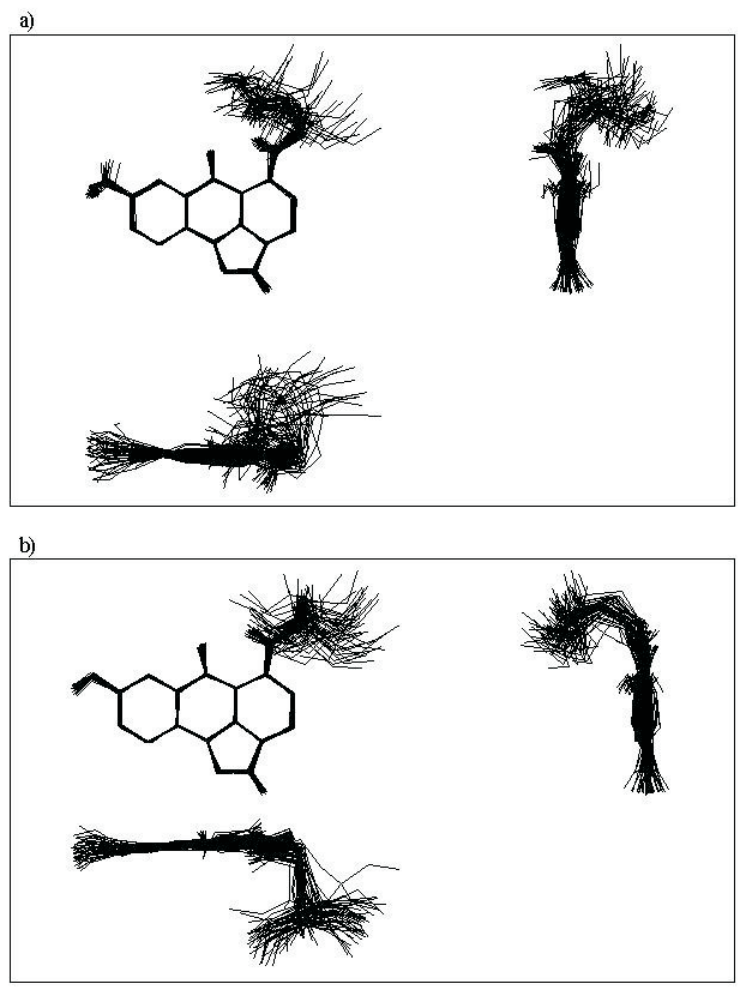

Figure 2. Orthogonal views of a composite of 40 snapshot geometries of C-1311: a) in water, and b) in the intercalation complex.

The acridine ring of the drug molecule in each snapshot has been fitted to its position in the first geometry. The dodecamer duplex, ions, and water molecules are omitted for clarity.

rical state of the $\mathrm{C} 5-\mathrm{NC} 5$ bond in water. The mean value of the torsion angle of this bond is $166.4 \pm 8.0^{\circ}$. This value differs significantly from $180^{\circ}$. As the result, the aromatic amino group in position 5 does not lie in the mean plane of the ring system. Moreover, the hydrogen bond between HNC5 and OC6 is not observed (Table 1).

The twist of the aromatic amino group facilitates formation of the hydrogen bonds by the atom $\mathrm{HN} 3+$ and stabilises the „closed“ conformation of the side chain.

The main difference occurs in the dynamic behaviour of the substituent in position 8 . Contrary to the hydroxyl group (C-1311), the methoxyl group is situated out of the mean plane of the ring system (Fig. 5c). Moreover, the methoxyl group could rotate around the bond $\mathrm{C} 8-\mathrm{OC} 8$ in water as well as in the inter-

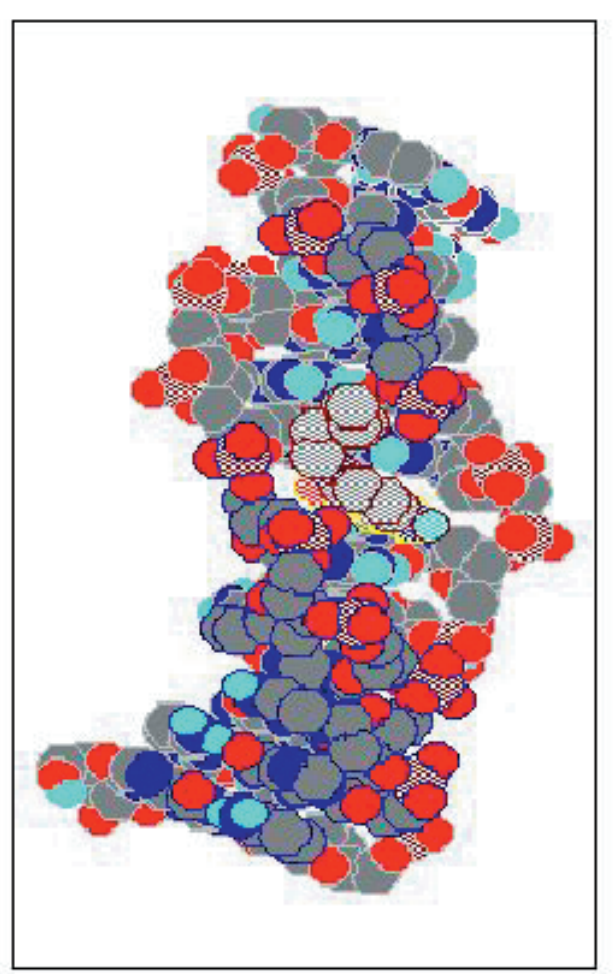

Figure 3. The representative geometry of the C-1311/dodecamer complex during simulation; view from the minor groove side.

The ions and water molecules are omitted for clarity.

calation complex. It seems that the rotation of the methoxyl group results from lack of water bridges which in the case of $\mathrm{C}-1311$ stabilise the orientation of the hydroxyl group.

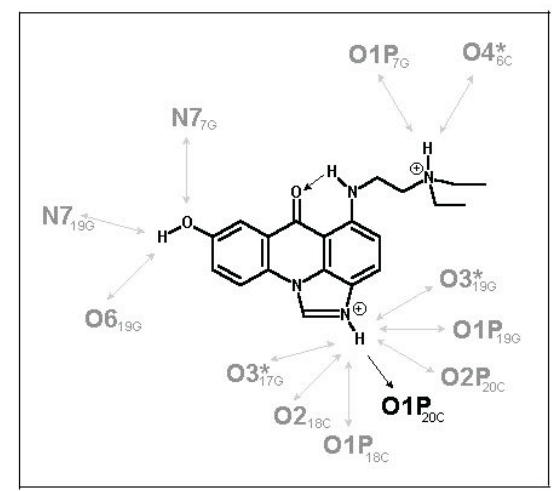

Figure 4. Hydrogen bonds (solid arrows) and water bridges (grey arrows) between the C-1311 molecule and the dodecamer observed during the simulation.

Only interactions which exist by more than $10 \%$ of the simulation time are presented. 
The similarity between the dynamic behaviour of C-1311 and C-1330 molecules is even higher in the intercalation complexes than in water (Fig. 1 and Fig. 5). The side chain lies out of the mean plane of the ring system as a result of the geometry of the NC5-C1+ bond. The side chain itself exists in „open“ conformation. Also formation of the characteristic hydrogen bond networks around $\mathrm{N} 3+$ and NR16 nitrogen atoms is similar for the two compounds in the intercalation complex. The rotation of the methoxyl group in position 8 observed during the simulation of C-1330 in water exists also when the molecule is involved in formation of the intercalation complex (Fig. 5c). This feature is the major difference between dynamic properties of C-1311 and $\mathrm{C}-1330$ in the complex with the dodecamer duplex.

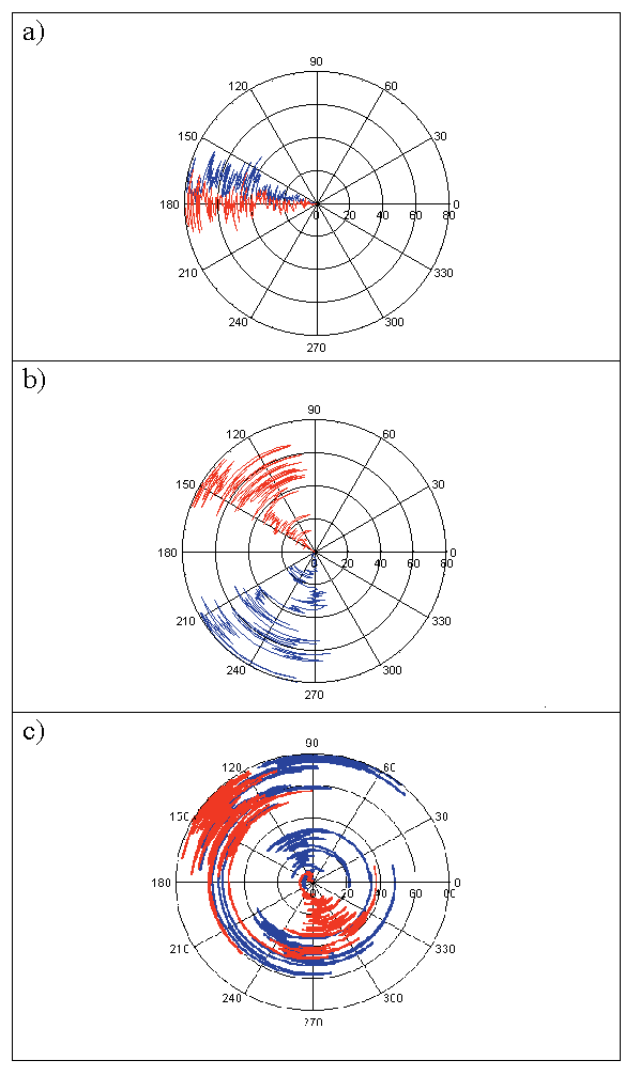

Figure 5. Histories of the torsion angles of selected bonds of C-1330: a) C5-NC5, b) NC5-C1+, c) $\mathrm{C8}-\mathrm{OC8}$.

Blue and red lines represent, respectively, values of the torsion angles in water and in the intercalation complex.

\section{C-1492}

The long side chain of the C-1492 molecule influences the dynamic properties of the whole molecule in water as well as in the complex.

In the two environments studied the aromatic amino group in position 5 is coplanar with the ring system (Fig. 6a). As the result, the hydrogen bond between HNC5 and OC6 is observed during more than half of the simulation time (Table 1). The lack of intramolecular hydrogen bonds in which atom HN5+ participates is a characteristic feature of the C-1492 trajectory in water (Table 1). This is due to a long distance between the terminal nitrogen atom and the ring system. In this situation the side chain exhibits large flexibility (Fig. 7a).

The flexibility of the side chain is significantly decreased after intercalation (Fig. 7b).

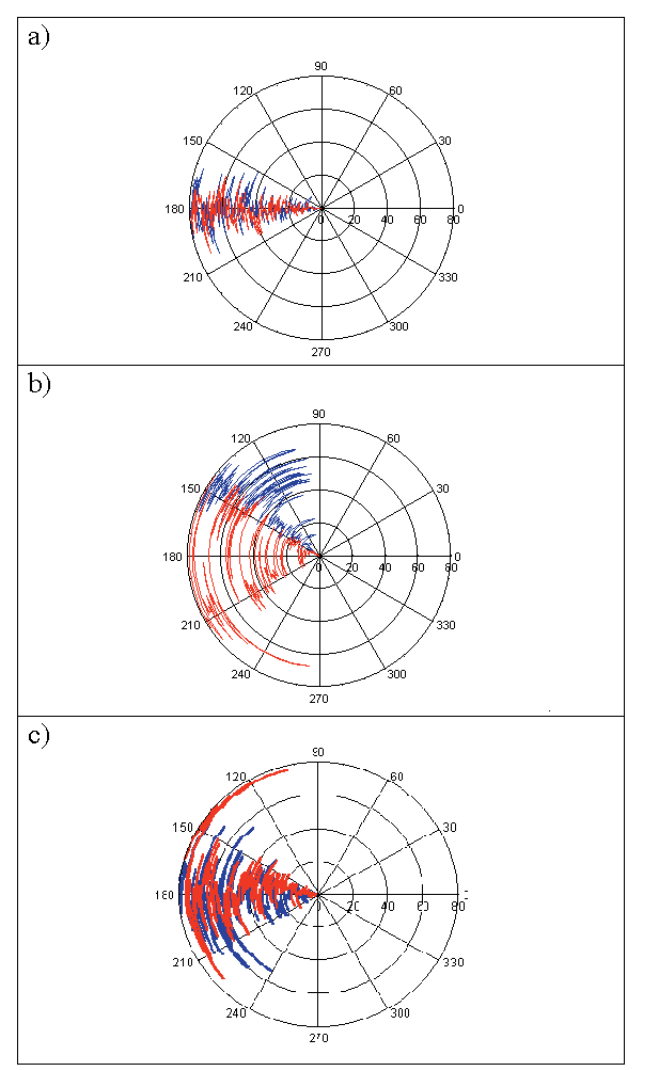

Figure 6. Histories of the torsion angles of selected bonds of C-1492: a) C5-NC5, b) NC5-C1+, c) C8-OC8.

Blue and red lines represent, respectively, values of the torsion angles in water and in the intercalation complex. 
Moreover, its orientation is significantly changed (Fig. 6b, and Fig. 7). In water the distal part of the side chain is nearly perpendicular to the ring system, while in the complex nearly the whole side chain is coplanar with the ring system.

The mean value of the torsion angle of the bond $\mathrm{C} 8-\mathrm{OC} 8$ does not differ from $180^{\circ}$ and a typical distribution of its values is observed (Fig. 6c).
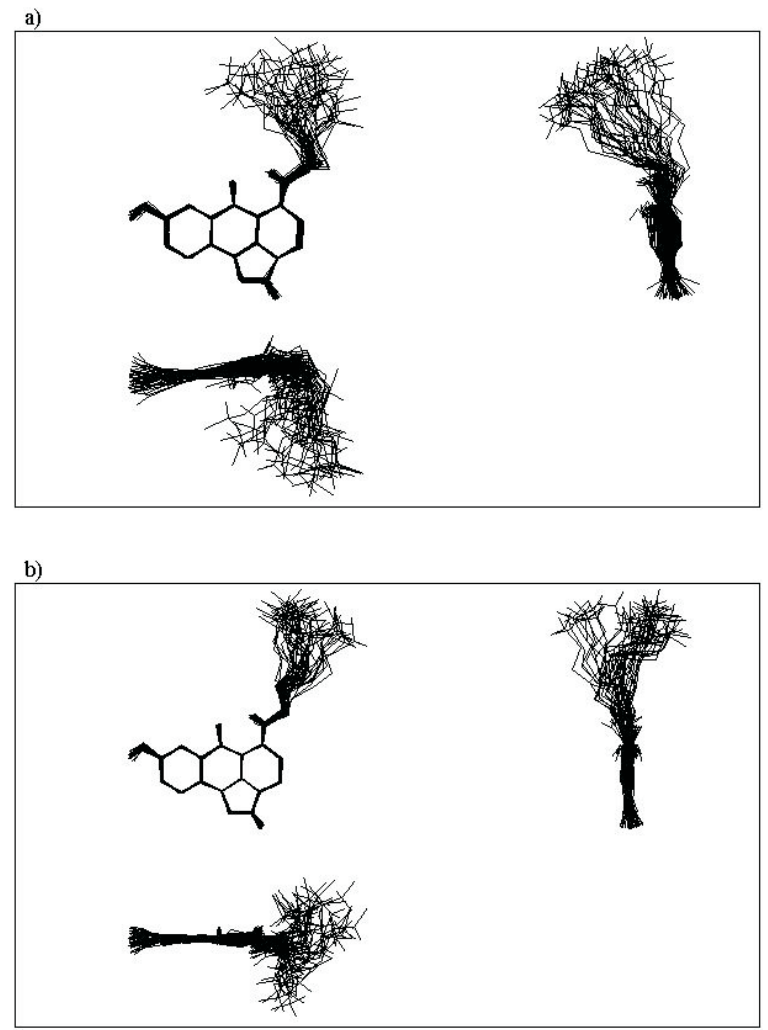

Figure 7. Orthogonal views of a composite of 40 snapshot geometries of C-1492: a) in water, and b) in the intercalation complex.

The acridine ring of the drug molecule in each snapshot has been fitted to its position in the first geometry. The dodecamer duplex, ions, and water molecules are omitted for clarity.

The length of the side chain affects in a specific manner the geometry of the intercalation complex. It is noteworthy that, in the case of C-1492 complex the protonated imidazole nitrogen does not form direct hydrogen bond with the dodecamer (Table 1). Only a few, rather unstable, water bridges between this fragment of the intercalator molecule and the dodecamer duplex have been detected.

The protonated aliphatic amino group of C-1492 interacts with the phosphate group of $7 \mathrm{G}$ by an electrostatic force as well as by a network of stable water bridges. The rest of the side chain is located near the mean plane of the ring system and out of the minor groove (Fig. 8). A more detailed analysis showed that the side chain may exist in two conformations

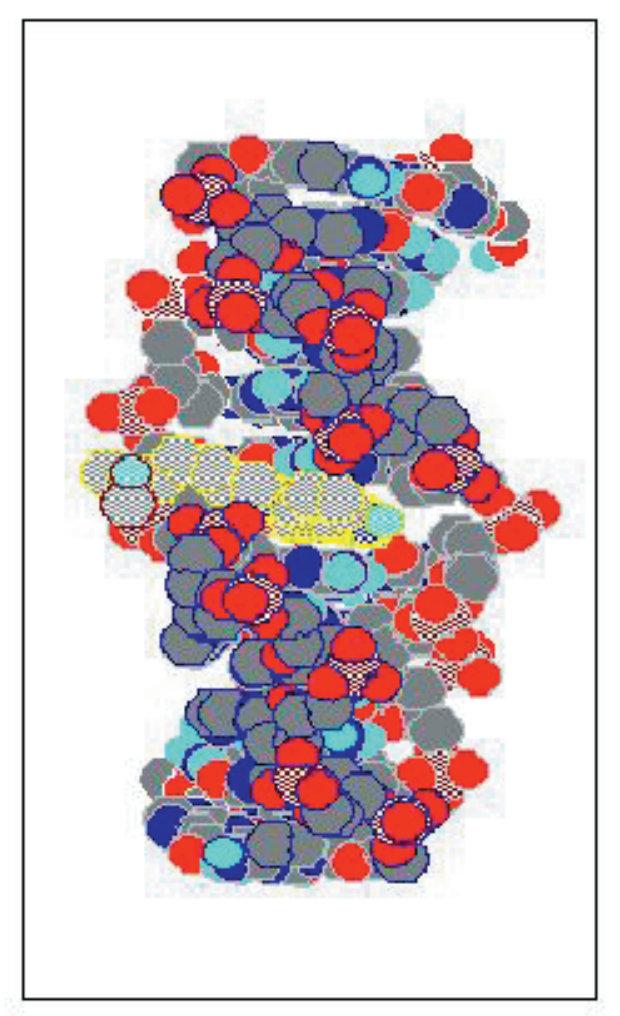

Figure 8. The representative geometry of the C-1492/dodecamer complex during simulation; view from the minor groove side.

The ions and water molecules are omitted for clarity.

determined by the geometrical state of the NC5-C1+ bond (Fig. 6b).

\section{C-1558}

We expected that substitution of the 8-OH group in compounds C-1311 by a t-butyl group in compound C-1558 should not introduce significant changes in the dynamic behaviour of the $N, N$-diethylaminoethyl side chain in wa- 
ter. However, the results obtained from the dynamic simulation were in contradiction to our expectations (Fig. 9).

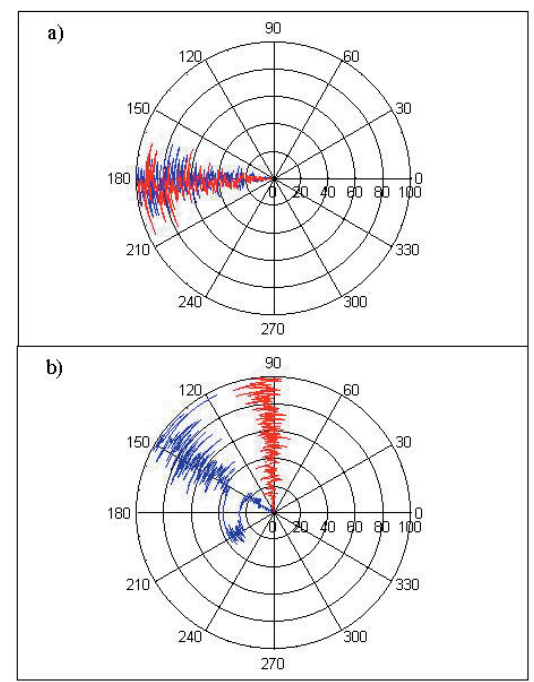

Figure 9. Histories of the torsion angle of selected bonds of C-1558: a) C5-NC5, b) NC5-C1+.

Blue and red lines represent, respectively, values of the torsion angles in water and in the intercalation complex.

In C-1558 the aromatic amino group is coplanar with the ring system (Fig. 9a), like in C-1311 and C-1492 in the same environment. The bond NC5-C1+ may exist in two geometrical states. These states are characterised by mean values of the torsion angle equal to about $150^{\circ}$ and $210^{\circ}$, respectively. This means that the side chain of $\mathrm{C}-1558$ in water deviates from the plane by the amino group of about $\pm 30^{\circ}$. It is noteworthy that:

i) this deviation is lower than for other IAs studied,

-ii) the side chain may spontaneously change the side of the ring system; such transition has never been observed for other IAs studied.

Together with the unexpected orientation of the side chain in $\mathrm{C}-1558$, one may also detect an atypical conformation of the side chain (Fig. 10). The formation of a network of hydrogen bonds between a protonated aliphatic amino group and aromatic nitrogen NC5 as well as carbonyl oxygen OC6 is a characteristic feature of $N, N$-diethylaminoethyl chain in water (C-1311 and C-1330), but is not observed for C-1558 (Table 1). As a result, the side chain of C-1558 does not form the typical "closed“ conformation. Only the hydrogen bond NC5-HNC5 $\rightarrow$ OC6 6 is observed in this system.

The geometry of C-1558 in the intercalation complex differs significantly from that observed in water mainly due to the state of NC5-C1+ bond (Fig. 9b and Fig. 10). In the in-
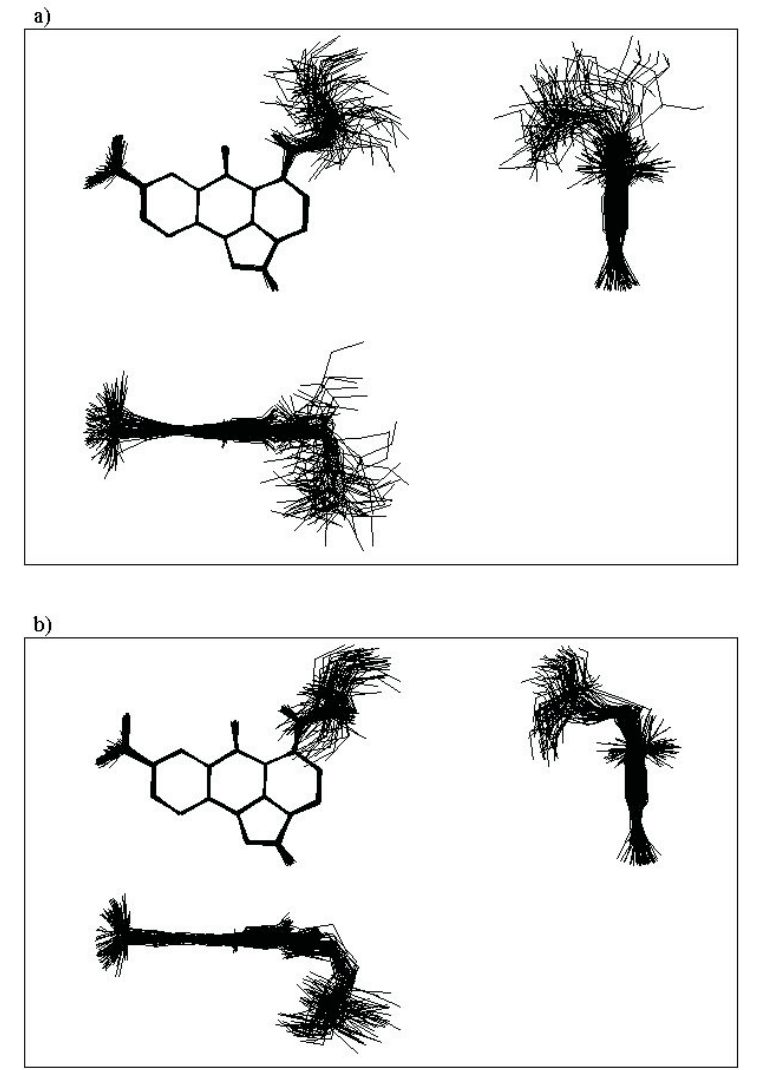

Figure 10. Orthogonal views of a composite of 40 snapshot geometries of C-1558: a) in water, and b) in the intercalation complex.

The acridine ring of the drug molecule in each snapshot has been fitted to its position in the first geometry. The dodecamer duplex, ions, and water molecules are omitted for clarity.

tercalation complex the side chain is perpendicular to the plane of the aromatic amino group. Moreover, this atypical geometrical state of the NC5-C1+ bond exhibits excellent stability during the whole simulation. This fact suggests that the orientation of the side 
chain might be stabilised by some additional interaction(s). The results presented in Table 1 indicate that an aliphatic amino group forms a hydrogen bond with the oxygen O3* $^{*}$ from $6 \mathrm{C}$. This interaction is observed during about $1 / 4$ of the simulation period. Unlike in the situation observed in the complexes containing C-1311 or C-1330, in the complex containing C-1558 the protonated aliphatic amino group does not interact with the phosphate groups by water bridges.

In the C-1558/dodecamer complex the hydrogen HNC5 (Scheme 2), participates in the intramolecular hydrogen bond with oxygen OC6 for less than $50 \%$ of the simulation time. Instead of that, it forms intermolecular bonds with sugar oxygen atoms from neighbouring bases (Table 1).

The analysis of water bridges between C-1558 and the dodecamer reveals additional peculiarities of this intercalation complex. The carbonyl oxygen OC6 is connected with phosphate oxygen $\mathrm{O} 2 \mathrm{P}$ and sugar oxygen $\mathrm{O} 4 *$ from $7 \mathrm{G}$ by the same water molecule (Fig. 11). Together with hydrogen bonds created by

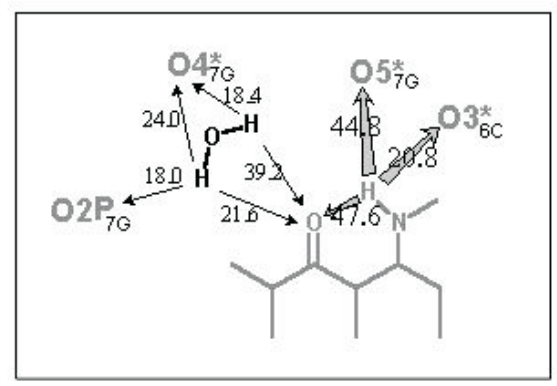

Figure 11. The characteristic network of hydrogen bonds formed around OC6 and HNC5 in C-1558/dodecamer complex.

Only hydrogen bonds existing by more than $10 \%$ of the simulation time are presented. The grey arrows represent direct hydrogen bonds between the drug and dodecamer. Numbers on the scheme indicate the percentage of the simulation time during which a particular hydrogen bond was observed.

HNC5, this water bridge participates in formation of a characteristic hydrogen bond network which is not observed for other IAs stud- ied. The participation of OC6 in the hydrogen bonds with water molecule strongly suggests that the geometry of intercalation complex of C-1558 differs from that of the complexes of IAs analysed up to now. Unusual stability of the hydrogen bond formed by HNR16 with O1P of 20C, Table 1, might support this suggestion.

\section{DISCUSSION}

The geometry of a C-1311 molecule in water only slightly differs from that observed in the crystal state (Fig. 1). The main difference refers to the orientation of the 8-OH group. The torsion angle $\mathrm{C} 7-\mathrm{C} 8-\mathrm{OC} 8-\mathrm{HOC} 8$ in the crystal state is $-3.4^{\circ}$. Similar values are observed at the beginning of the simulation. However, after less than $20 \mathrm{ps}$ of the main simulation the geometrical state of the C8-OC8 bond flipped over to values around $180^{\circ}$. This orientation of the 8-OH group was then stable during the rest of the simulation time. The same orientation of this group was observed during simulation of C-1492. This might suggest that the geometrical state of the $\mathrm{C} 8-\mathrm{OC} 8$ bond found in the crystal state results from specific intermolecular interactions in the crystal lattice.

The results of molecular dynamics simulation presented in this paper indicate that conformation of IAs side chain in water, as well as its orientation in relation to the ring system depends not only on the chemical constitution of the chain, but also on the substituent in position 8 . The aromatic amino group attached in position 5 is coplanar to the ring system in the case of C-1311 (8-OH), C-1492 (8-OH), and $\mathrm{C}-1558$ (8-tBu), but is twisted by about $14^{\circ}$ in the case of C-1330 (8-OMe). The $N, N$-diethylaminoethyl chain exhibits a tendency to form a "closed" conformation when hydroxyl (C-1311) or methoxyl group (C-1330) is attached in position 8 . However, this tendency is not observed when a bulky t-butyl group (C-1558) occupies this position. Reasons for 
this behaviour are not clear yet. One may suggest that the polar oxygen atom situated in position 8 could create the hydration shell around the IA molecule with such properties that intramolecular hydrogen bonds are more favoured than intermolecular ones. However, the presence of a heavy and bulky t-butyl group also could change the dynamics of the IA molecule in such a manner that "closed" conformation of the side chain would be dynamically unstable. Atypical dynamics of the torsion angle of NC5-C1+ bond observed for C-1558 (Fig. 9b) seems to support this hypothesis.

The intercalation of the IA molecule into the DNA fragment significantly changes the geometry of the side chain. The $N, N$-diethylaminoethyl chain exhibits a strong tendency to fit into the minor groove (Fig. 3). The observed changes in the conformation, as well as the orientation of this chain, result from this tendency. The side chain fits to the groove due to at least four types of interactions:

-i) electrostatic interaction between positively charged $\mathrm{N} 3^{+}$atom and negatively charged phosphate groups,

-ii) van der Waals interactions between side chain and atoms on the surface of the groove,

- iii) direct hydrogen bonds in which hydrogen $\mathrm{HN}^{+}+$plays the role of donor and polar, oxygen or nitrogen, atoms from phosphate/sugar skeleton or bases play the role of acceptors,

-iv) „water bridges“ between the nitrogen cation and oxygen atoms bearing partial negative charge.

Only the last two types of interactions could be detected during the analysis of the dynamic trajectory. The water bridges stabilising the position of $N, N$-diethylaminoethyl chain were found in the intercalation complexes formed by C-1311 and C-1330 (Fig. 4). It is interesting to note that the bridges were not observed in the complex formed by C-1558. Instead, a direct hydrogen bond between side chain and sugar oxygen of $6 \mathrm{C}$ was found (Table 1). On the contrary, direct hydrogen bonds were not detected in complexes formed by C-1311 and C-1330. The $N, N$-diethylaminoethyl chain is relatively short and its direct as well as indirect interactions with atoms forming the minor groove were limited to the neighbouring base-pairs only.

Our previous results obtained for such anthracenediones as mitoxantrone or ametantrone [17] showed, that a long enough polar chain could interact with atoms from at least two base-pairs from each side of the intercalation place. We expected that a similar range of interactions should be detected in the case of long $N, N$-dimethylaminopentyl chain of C-1492. However, this chain did not exhibit any tendency to fit into the minor groove (Fig. 8). This observation indicates that some repulsive forces exist between the relatively long, strongly apolar aliphatic chain and strongly polar interior of the minor groove. In such situation, long-range attractive electrostatic forces move the protonated aliphatic amino group to the vicinity of negatively charged phosphate groups. The flexible pentyl chain could adopt two optimal conformations in which electrostatic interactions were as strong as possible (Fig. 7b). In the two conformations the whole side chain was placed near the mean plane of the ring system.

The relative orientation of the IAs in the intercalation complexes was also depended on the substituent in position 8 and the chemical constitution of the side chain in position 5 (Fig. 12). For the drugs C-1311 and C-1330, the compounds possessing a polar substituent in position 5 and a relatively short side chain which fits into the minor groove, the acridine ring system was parallel to the neighbouring base pairs, but the imidazole ring was inclined with respect to this plane. A different situation was observed in the C-1558/dodecamer complex. The bulky and apolar substituent attached in position 8 could work as an anchor which limited the movements and stabilised the particular orientation of the drug molecule inside the intercalation cavity. The high 


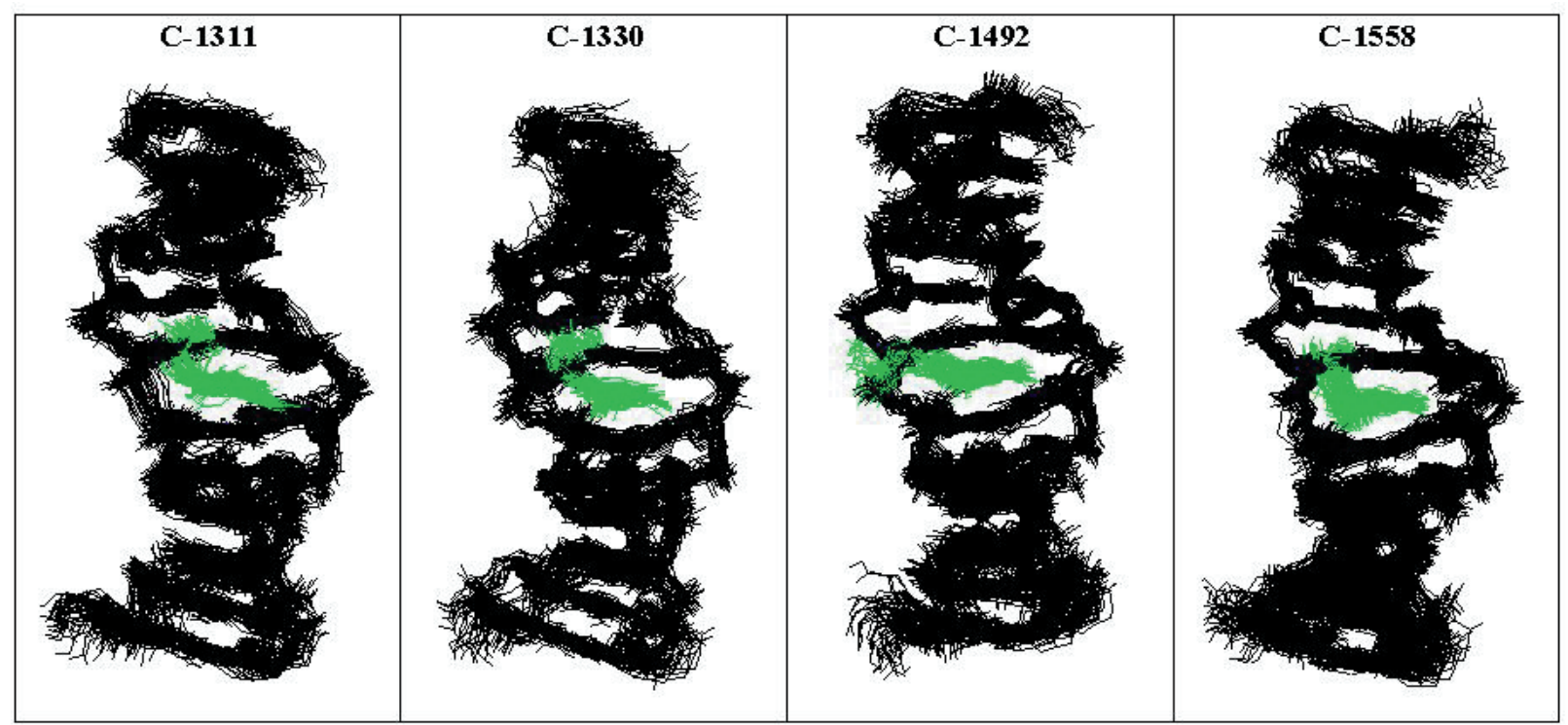

Figure 12. Composites of 40 snapshot geometries of IA/dodecamer complexes.

Views from the minor groove side. The sugar $\mathrm{C} 1^{*}$ atoms of 8 central base-pairs in each snapshot have been fitted to their positions in the first snapshot. The ions and water molecules are omitted for clarity.

level of interactions between the dodecamer and the acridinone oxygen, as well as the aromatic amino group in position 5 is the characteristic feature of this orientation. It is noteworthy that presence of two t-butyl substituents in positions 2 and 7 of a proflavine molecule makes the intercalation impossible [22]. The 2,7-di-t-butylproflavine interacts with DNA but does not intercalate into it. Results of biochemical experiments, which were done in our Department, indicated, however, that C-1558 was able to intercalate into DNA (Dzięgielewski, J., Ślusarski, B., Konitz, A., Składanowski, A. \& Konopa, J., unpublished).

It seems that long, flexible, and apolar chain present in C-1492 has no influence on the orientation of the ring system inside the intercalation cavity. The mean plane of the ring system is practically parallel to the base-pairs. As a result of such "free" orientation of the intercalator ring system no hydrogen bond or water bridges between protonated imidazole nitrogen and the dodecamer are observed.

The substituent present in position 8 of the imidazoacridinone ring system affects a number of structural and dynamic properties of the molecules in water, as well as in the inter- calation complex. We found that this substituent affects at least:

$\checkmark$ i) orientation of the aromatic amino group in water,

ii) geometrical state of the $\mathrm{NC} 5-\mathrm{C} 1+$ bond; the influence is higher in water than in the complex,

-iii) conformation of the side chain in water,

iv) geometry of the intercalation complex,

$\checkmark v$ type and dynamics of the interaction between the drug and the dodecamer duplex.

This observation seems to be the main conclusion which can be derived from the results presented in this work.

\section{R E F E R E N C E S}

1. Cholody, M.W., Martelli, S., Łukowicz, J. \& Konopa, J. (1990) 5-[(Aminoalkyl)amino]-imidazo[4,5,1-de]acridin-6-ones as a novel class of antineoplastic agents. Synthesis and biological activity. J. Med. Chem. 33, 49-52.

2. Cholody, M.W., Martelli, S. \& Konopa, J. (1992) Chromophore-modified antineoplastic imidazoacridinones. Synthesis and activity 
against murine leukemias. J. Med. Chem. 35, 378-382.

3. Cholody, M.W., Horowska, B., ParadziejŁukowicz, J., Martelli, S. \& Konopa, J. (1996) Structure-activity relationship for antineoplastic imidazoacridinones. Synthesis and antileukemic activity in vitro. J. Med. Chem. 39, 1028-1032.

4. Mazerska, Z., Augustin, E., Składanowski, A., Bibby, M.C., Double, J.A. \& Konopa, J. (1998) C-1311. Drugs Future 23, 702-706.

5. Burger, A.M., Double, J.A., Konopa, J. \& Bibby, M.C. (1996) Preclinical evaluation of novel imidazoacridinone derivatives with potent activity against experimental colorectal cancer. Brit. J. Cancer 74, 1369-1374.

6. Dzięgielewski, J., Składanowski, A. \& Konopa, J. (1996) Noncovalent binding of potent antitumor imidazoacridinones to DNA. Ann. Oncol. 7 (Suppl. 1), 56.

7. Dzięgielewski, J. \& Konopa, J. (1996) Interstrand crosslinking of DNA induced in tumor cells by a new group of antitumor imidazoacridinones. Proc. Amer. Assoc. Cancer Res. 37, Abstract 2800.

8. Dzięgielewski, J., Mazerska, Z. \& Konopa, J. (1998) Enzymatic activation of antitumor imidazoacridinone drug, C-1311, in relation to its DNA interaction. Exp. Toxicol. Pathol. 50, 101.

9. Cieplak, P., Rao, S.N., Grootenhuis, P.D.J. \& Kollman, P.A. (1990) Free energy calculations on base specificity of drug-DNA interactions: Application to daunomycin and acridine intercalation into DNA. Biopolymers 29, 717-727.

10. Swaminathan, S., Beveridge, D.L. \& Berman, H.H. (1990) Molecular dynamics simulation of a deoxynucleotid-drug complex: d(CpG)/ proflavine. J. Phys. Chem. 94, 4660-4665.

11. Langley, D.R., Dolye, T.W. \& Beveridge, D.L. (1991) The dyneimicin-DNA intercalation complex. A model based on DNA affinity cleav- age and molecular dynamics simulations. $J$. Am. Chem. Soc. 113, 4395-4403.

12. Herzyk, P., Neidle, S. \& Goodfellow, J.M. (1992) Conformation and dynamics of drug-DNA intercalation. J. Biomol. Struct. Dyn. 10, 97-139.

13. Gallego, J., Ortiz, A.R. \& Gago, F. (1993) A molecular dynamics study of the bis-intercalation complex of echinomycin with $\mathrm{d}(\mathrm{ACGT})_{2}$ and $\mathrm{d}(\mathrm{TCGA})_{2}$ : Rationale for sequence-specific Hoogsteen base pairing. J. Med. Chem. 36, 1548-1561.

14. Denny, B.J., Wheelhouse, R.T., Stevens, M.F.G., Tsang, L.L.H. \& Slack, J.A. (1994) NMR and molecular modeling investigation of the mechanism of activation of the antitumor drug Temozolomide and its interaction with DNA. Biochemistry 33, 9045-9051.

15. Van Garderen, C.J. \& Van Houte, L.P.A. (1994) The solution structure of a DNA duplex containing the cis-Pt( $\left.\mathrm{NH}_{3}\right)_{2}\left[\mathrm{~d}(-\mathrm{GTG}-)-N_{7}(\mathrm{G})\right]$ adduct, as determined with high-field NMR and molecular mechanics/dynamics. Eur. J. Biochem. 225, 1169-1179.

16. Bear, B. \& Remers, W.A. (1996) Computer simulation of the binding of amonafide and azonafide to DNA. J. Comput.-Aided Molec. Design 10, 165-175.

17. Mazerski, J., Martelli, S. \& Borowski, E. (1998) The geometry of intercalation complex of antitumor mitoxantrone and ametantrone with DNA: Molecular dynamics simulations. Acta Biochim. Polon. 45, 1-11.

18. VanGusteren, W.F., Billeter, S.R., Eising, A.A., Hunenberger, P.H., Kruger, P., Mark, E.A., Scott, W.R.P. \& Tiron, I.G. (1996) Biomolecular Simulation: GROMOS96 Manual and User Guide. vdf Hochschulverlag AG, Zurich.

19. Ryckeart, J.P., Ciccotti, G. \& Berendsen, J.H.C. (1977) Numerical integration of the cartesian equations of motion of a system with 
constraints: Molecular dynamics of n-alkanes. J. Comput. Phys. 23, 327-341.

20. Subraminian, P.S., Ravishanker, G. \& Beveridge, D.L. (1991) Molecular dynamics of B-DNA including water and counterions. A 140-ps trajectory for d(CGCGAATTCGCG) based on the GROMOS force field. J. Am. Chem. Soc. 113, 5027-5040.

21. VanGunsteren, W.F., Berendsen, H.J.C., Geursten, R.G. \& Zwinderman, H.R.J. (1986)
A molecular computer dynamics simulation of an eight-base-pair DNA fragment in aqueous solution: Comparison with experimental 2D NMR data. Ann. N. Y. Acad. Sci. 482, 287303.

22. Müller, W., Crother, D.M. \& Waring, M.J. (1973) A non-intercalating proflavine derivative. Eur. J. Biochem. 39, 223-234. 